\title{
SERVIÇO DE REFERÊNCIA NACIONAL EM FILARIOSES: ACREDITAÇÃO E GARANTIA DA QUALIDADE
}

Eduardo Brandão, Patrícia dos Santos Silva, Paulo Sérgio Ramos de Araújo, Zulma Medeiros, Ana Maria Aguiar Santos, Yara Miranda Gomes e Abraham Rocha

\section{RESUMO}

O Serviço de Referência Nacional em Filarioses (SRNF) do Centro de Pesquisas Aggeu Magalhães, Fiocruz, desenvolve atividades multi e interdisciplinares no controle e eliminação da filariose bancroftiana. A fim de melhorar a qualidade dos serviços oferecidos e a segurança do paciente, foi implantado um sistema de qualidade baseado nos padrões de qualidade propostos pela Joint Commission International (JCI). Esta nota tem como objetivo descrever a implantação do programa de cuidados ambulatoriais na referida unidade para outorga da certificação. O processo foi iniciado com um estudo minucioso do Manual de Cuidados Ambulatoriais, realizando-se, em seguida, a elaboração da documentação pertinente, a conscientização e capacitação dos recursos humanos, a adequação física das instalações e, posteriormente, a avaliação externa pela equipe de auditores do JCI. A instituição do novo sistema de qualidade evidenciou que o comprometimento da gestão e de toda a equipe foi, sem dúvida, o elemento mais importante para o alcance das metas e sucesso das ações. Este processo viabilizou avanços efetivos na melhoria da qualidade dos serviços prestados e da segurança do paciente, não somente no SRNF como em toda a instituição na qual ele se encontra inserido.

DESCRITORES: Ambulatório; gestão da qualidade; certificação; filariose linfática. 1 Serviço de Referência Nacional em Filarioses, Centro de Pesquisas Aggeu Magalhães, Fundação Oswaldo Cruz, Ministério da
Saúde, Recife-PE, Brasil.

Endereço para correspondência: Eduardo Brandão. Serviço de Referência Nacional em Filarioses, Centro de Pesquisas Aggeu Magalhães, Fundação Oswaldo Cruz, Ministério da Saúde, Avenida Professor Moraes Rego S/N, CEP 50670-420, Recife, PE, Brasil. E-mail: brandaoe@cpqam.fiocruz.br 


\section{ABSTRACT}

National centre of lymphatic filariasis: accreditation and quality assurance

The National Reference Service for filariasis (SRNF), the Center for Research Aggeu Magalhães - FIOCRUZ, develops multi and interdisciplinary activities in control and elimination of lymphatic filariasis. In order to improve the quality of services offered, as well as patient safety, a quality system based on the quality standards proposed by the Joint International Commission (JCI) has been implemented. Given these considerations, the present note aimed to describe the implementation of an ambulatory healthcare program at the SRNF for the granting of certification. Thus, the Ambulatory Healthcare Manual was studied. The process began with a thorough study of the Ambulatory Care Manual. Then followed the preparation of pertinent documentation, enhancing staff awareness and skills, and physically adjusting the facilities and further external evaluation by the team of JCI's auditors. The quality implementation process showed that the engagement of the institution's managers and staff was clearly the most important factor for project success, and the initiative to implement the program improved quality and patient safety not only at the SRNF but also the institution to which it belongs.

KEY WORDS: Ambulatory; quality management; certification; Lymphatic Filariasis.

O Serviço de Referência Nacional em Filarioses (SRNF) do Centro de Pesquisas Aggeu Magalhães, Fiocruz, é o único serviço de referência no Brasil relacionado à filariose linfática provocada pela Wuchereria bancrofti. Fundado em 1986 como um grupo de trabalho em filarioses, sua produção de conhecimentos técnicos e científicos ao longo de mais de duas décadas deu suporte ao Ministério da Saúde (MS) para credenciar suas atividades como referência no ano de 2002 (Buss \& Gadelha, 2002). Em março de 2008, o SRNF foi submetido a uma auditoria externa proposta pela Coordenação Geral de Laboratórios do MS de acordo com as exigências da Portaria Ministerial $\mathrm{n}^{\circ}$ 70 , sendo reabilitado como Serviço de Referência por meio da Portaria ${ }^{\circ} 97$ de 23 de outubro de 2008 (MS 2004; 2008).

Com a implementação do Programa Nacional de Eliminação da Filariose Linfática (PNEFL), o SRNF vem assessorando as áreas que estão sob impacto do tratamento em massa com a dietilcarbamazina no acompanhamento de casos positivos e apoio ao plano de morbidade filarial. Além disso, o serviço está comprometido com o PNEFL na reavaliação dos focos que, desde a década de 1960, têm sido considerados extintos na busca por certificação da eliminação (Rocha et al., 2009).

Desde seu credenciamento até a atualidade, muitos avanços foram alcançados pelo SRNF. Um sistema de qualidade baseado nos padrões de acreditação propostos pela Joint Commission International (JCI) foi implantado (JCI, 2010) a fim de melhorar a qualidade dos serviços oferecidos e a segurança do paciente. Diante do exposto, o objetivo desta nota é descrever o processo de implantação do programa de cuidados ambulatoriais na referida unidade para outorga da certificação. 
A implantação do sistema de qualidade baseado nos padrões de acreditação da JCI para obtenção da certificação foi dividida em quatro etapas: a) introdução da metodologia por meio da apresentação dos padrões e elementos de mensuração que compõem o manual de Cuidados Ambulatoriais pela equipe de consultores do Consórcio Brasileiro de Acreditação (CBA); b) visita diagnóstica para verificação e aplicação prática dos padrões do manual; c) visita de educação para avaliar a evolução das ações implantadas, fortalecer os processos que se encontravam em andamentos e preparar a instituição para a visita oficial de certificação; d) visita de certificação para verificar a adequação de todos os padrões constantes do manual.

A avaliação diagnóstica realizada em 2010 para a verificação dos 11 padrões aplicáveis demonstrou que o SRNF possuía, naquele momento, $68 \%$ de conformidade. Foram considerados como potencialidades os seguintes padrões: Prontuário do paciente e fluxo de informações (PRI), Acesso e avaliação do paciente (PAA) e Governo e liderança (GAL), sendo observados percentuais de conformidades de $98 \%$, 97\% e $96 \%$, respectivamente. No entanto, padrões considerados essenciais para o êxito do processo como, por exemplo, Educação de pacientes e familiares (PFE), Melhoria da qualidade e segurança do paciente (IQS), Controle de infecções e segurança das instalações (IFS) e Direitos e responsabilidades do paciente (PRR) foram evidenciados como fragilidades, pois demonstravam percentuais de não conformidade acima de $40 \%$. Essas não conformidades estavam associadas com a falta de planejamento da educação de pacientes e familiares, ausência de um programa de qualidade que possibilitasse ações de melhoria contínua da qualidade, inexistência de um programa de redução de riscos de infecções e de um manual institucional que definisse o direito dos pacientes e familiares.

Transcorridos, aproximadamente, 18 meses após a avaliação diagnóstica, a visita de certificação demonstrou uma redução significativa do percentual de situações parcialmente conformes e não conformes. Isso conferiu ao Serviço de Referência Nacional em Filarioses a outorga da acreditação, o que o tornou o primeiro serviço relacionado a doenças tropicais a receber esta certificação no mundo. Somente uma não conformidade foi evidenciada, esta se referia ao padrão PRI e estava associada à completitude da lista do resumo, da qual constavam, exclusivamente, informações referentes aos diagnósticos significativos, excluindo dados referentes a alergias a medicamentos e a quaisquer procedimentos cirúrgicos e internações anteriores.

Processo complexo, a implantação da qualidade no ambulatório do SRNF teve como principal barreira o estabelecimento da cultura de qualidade na força de trabalho, evidenciada pela resistência apresentada pela equipe que, naquele momento, não foi capaz de identificar os ganhos que o processo poderia gerar. Este fato também foi evidenciado por Ferreira e Aguiar durante o processo de implantação do Programa de Cuidados Clínicos em uma das unidades do Instituto Nacional de Câncer (Ferreira \& Aguiar, 2012). Superado 
este obstáculo, muitos avanços foram alcançados, principalmente no que se refere ao programa de melhoria da qualidade por meio da implantação da gestão de indicadores, os quais permitiram uma avaliação mais precisa dos processos desenvolvidos pela instituição. Dentre os indicadores avaliados, o que se referia à completitude das informações clínicas foi um dos que apresentaram maior impacto, pois permitiu aumentar a segurança do paciente e otimizar a dinâmica de cuidados. Outro indicador que também permitiu grandes avanços no processo de cuidado dizia respeito ao tempo oportuno para a liberação de laudos, sendo estabelecido o prazo máximo de 15 dias para a realização das análises e liberação dos resultados.

Um estudo realizado em uma unidade ambulatorial de cuidado clínico em insuficiência cardíaca apontou que a gestão de indicadores de desempenho de um programa clínico e sua proficiência interna e externa são atividades fundamentais para garantir o sucesso das ações de melhoria da qualidade e segurança do paciente (Pavini et al., 2013). Esse estudo apontou ainda que a acreditação de uma unidade ambulatorial e a posterior certificação dos programas de cuidados clínicos contribuem significativamente para uma melhor evolução dos resultados desses indicadores.

Quando se analisa a logística da acreditação do ponto de vista do gerenciamento da unidade de cuidados, também são notáveis as mudanças que esse processo desencadeia. Significativas melhorias foram alcançadas em relação a uma melhor integração e sistematização da gestão, o que possibilitou efetivar o mapeamento, a formalização e a melhoria contínua dos processos. Diante de um novo modelo de gestão e com a introdução de uma metodologia baseada na resolução de problemas por meio de reuniões de análises críticas, vários problemas foram solucionados, o que viabilizou o processo de certificação.

Pode-se concluir que são notórios os benefícios alcançados pela implantação do processo de acreditação, dentre os quais podemos citar a elevação da confiança da população quanto aos seguintes aspectos: segurança e qualidade do cuidado, construção de um ambiente de trabalho seguro e eficiente que contribui para a satisfação do trabalhador, desenvolvimento de uma assistência focada em escutar o paciente e seus familiares com respeito a seus direitos no estabelecimento do processo de cuidado e estabelecimento de um estilo de liderança colaborativa que define prioridades e assegura a liderança contínua dos processos de qualidade e segurança do paciente em todos os níveis.

O processo de implementação do novo sistema de qualidade evidenciou que o comprometimento da gestão e de toda a equipe foi, inegavelmente, o elemento mais importante para o alcance das metas e sucesso das ações. Este processo viabilizou avanços efetivos na melhoria da qualidade dos serviços prestados e da segurança do paciente, não somente no SRNF, mas em toda a instituição na qual ele se encontra inserido. 


\section{REFERÊNCIAS}

1.Buss PM, Gadelha P. Fundação Oswaldo Cruz: experiência centenária em biologia e saúde pública. SP Perspectiva 16: 73-83, 2002.

2.Ferreira RS, Aguiar B. Implantação do Programa de Cuidados Clínicos, proposto pela Joint Comission International, em um Centro de Transplante de Medula Óssea de um Hospital de Câncer. Rev Acreditação 2: 17-29, 2012

3.Joint Commission International. Accreditation Standards for Ambulatory Care Guideline. Joint Commission Resources, Illinois, United States, 2010.

4.MS. Ministério da Saúde. Secretaria de Vigilância em Saúde (2004) Portaria nº 70, 23.12.2004. Brasília.

5.MS. Ministério da Saúde. Secretaria de Vigilância em Saúde (2008) Portaria nº 97, 23.10.2008. Brasília.

6.Pavini AE, Pinto CMN, Trindade CER. Processo de certificação do programa ambulatorial de cuidado clínico de insuficiência cardíaca e o impacto nos indicadores de desempenho. Rev Acreditação 3: 27-32, 2013.

7.Rocha A, Barbosa CS, Brandão Filho SP, Oliveira CMF, Almeida AMP, Gomes YM. Primeiro workshop interno dos serviços de referência do Centro de Pesquisas Aggeu Magalhães da Fundação Oswaldo Cruz. Rev Soc Bras Med Trop 42: 228-234, 2009. 